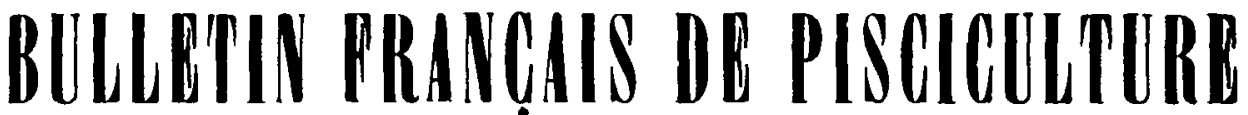

\section{LA PISCICUlTURE DU TILAPIA}

\author{
par B. CHARPY \\ Inspecteur des Eaux et Forêts de la F. O. M., \\ chargé de la Pisciculture en A. E. F.
}

Depuis quelques années, la pisciculture du Tilapia prend de plus en plus d'importance en Afrique Centrale; c'est que les responsables de ces immenses territoires cherchent les moyens les plus efficaces et les plus rapides pour pallier la sous-alimentation des populations africaines, l'une des causes importantes de la lenteur du développement économique de ces régions par suite du mauvais rendement de la maind'œuvre. Les hygiénistes et les médecins ont remarqué que si ces populations étaient sous-alimentées, leur ration journalière présentait surtout un déficit important en protéines d'origine animale. Au Moyen-Congo, au Gabon et dans une grande partie de l'Oubangui, le manioc et la banane forment l'essentiel de leur nourriture parce qu'il est très difficile de se procurer viande et poisson.

L'élevage et tout au moins l'élevage bovin est extrêmement difficile dans un pays où les pâturages sont très pauvres et où de nombreuses maladies déciment les troupeaux. La viande de chasse se raréfie de plus en plus et on cherche actuellement à préserver le capital qui subsiste. Les côtes marines, certains fleuves, certains lacs sont très poissonneux et sont exploités par des populations de pêcheurs, mais le transport du poisson jusqu'au fond de la brousse demande une préparation délicate et son prix de revient est tel que la plupart des villageois n'ont pas les moyens financiers suffisants pour l'acheter.

Ce sont les Belges qui, les premiers, vers la fin de la dernière guerre, ont pensé à la pisciculture comme source de poisson pour l'alimentation des populations qui avaient considérablement augmenté dans les régions minières de Katanga autour d'Élisabethville. Les premières investigations faites dans la faune locale avaient permis de sélectionner deux Cichlidae indigènes, Tilapia macrochir et Tilapia melanopleura qui paraissaient pouvoir s'élever parfaitement en étang de Pisciculture.

En 1946, fut créée, à Élisabethville, une station de recherche piscicole dont l'une des premières tâches fut de préciser l'utilisation du Tilapia en pisciculture. Après M. HuEt, le premier grand technicien du Tilapia, fut le Dr. A. F. DE BONT, actuellement Directeur de la station de recherche piscicole du Congo belge, dont les nombreuses publications sont à la base de tout ce qui a été entrepris en Afrique Centrale. 
Dès que l'effectif du personnel des Eaux et Forêts l'a permis, l'Afrique Équatoriale Française s'est intéressée à cette question en créant près de Brazzaville la station de pisciculture de la Djoumouna (fig. 1). Son premier et unique rôle jusqu'ici a été de mettre au point, sous le climat de l'A. É. F., une méthode de pisciculture rationnelle et efficiente qui puisse être offerte non seulement aux colons et gros exploitants à la recherche d'un appoint de nourriture pour leurs travailleurs, mais aussi aux paysans africains des villages les plus reculés pour qu'ils produisent eux-mêmes le complément de nourriture nécessaire à leur bonne alimentation. Il convenait donc de rechercher une méthode simple, facile à faire appliquer, basée sur des notions aussi précises et concises que possible, en se rappelant que nos futurs pisciculteurs n'avaient jamais envisagé jusqu'ici la possibilité de cultiver du poisson.

Le but des notes qui vont suivre est d'exposer les résultats acquis à la station de pisciculture de la Djoumouna depuis qu'elle fonctionne comme station de recherches, c'est-à-dire depuis le 1er Janvier 1953. L'application a suivi rapidement l'obtention des premiers résultats puisqu'il existe déjà près de 3.000 étangs dans la Fédération, l'immense majorité étant constituée par de petits bassins de pisciculture familiale construits et exploités par des Africains.

Mais nous voulions auparavant placer le problème dans son vrai cadre, qui est très différent de celui de la pisciculture dans les pays tempérés : il ne s'agit pas de repeupler les rivières, il ne s'agit pas, pour l'instant tout au moins, de fabriquer de beaux poissons, il s'agit avant tout de faire du kg. de poisson aussi rapidement que possible et avec les faibles moyens dont dispose le paysan africain.

Nous diviserons notre exposé en deux parties :

$1^{\circ}$ Le Tilapia : ses caractères spécifique et sexuel, sa croissance, sa nourriture;

$2^{\circ}$ L'exploitation des étangs de pisciculture à Tilapia.

\section{LE TILAPIA}

Tilapia macrochir et Tilapia melanopleura, les deux seules espèces utilisées couramment jusqu'ici en pisciculture, possèdent, comme tous les Cichlidae, une nageoire dorsale très développée, continue, dont la partie antérieure épineuse n'est pas séparée de la partie molle; ils ont des dents sur les mâchoires et sur les os pharingiens. Le genre Tilapia est caractérisé par des dents disposées en plusieurs rangées (celles de la rangée externe bicuspides, celles de la rangée interne tricuspides) et par l'absence de canines sur le devant.

Caractòres spócifiques. - La reconnaissance des différentes espèces de Tilapia est très délicate; elle est basée en partie sur le nombre de branchiospines du premier arc branchial : Tilapia macrochir en possède 22 à 24, Tilapia melanopleura 8 à 12. Celles de Tilapia melanopleura sont beaucoup plus grosses et plus courtes que celles de Tilapia macrochir; de sorte qu'en pisciculture où ne sont utilisées que ces deux espèces, 
on les distingue facilement en soulevant l'opercule et en laissant retomber les feuillets des branchies.

Le nombre et la forme des branchiospines, par le filtre qu'elles constituent, donnent une indication sur le régime alimentaire ; c'est ainsi qu'on peut prévoir a priori que Tilapia macrochir sera plus microphage que Tilapia melanopleura.

Un nombre élevé de branchiospines semble également caractériser les espèces qui pratiquent l'incubation buccale comme Tilapia macrochir.

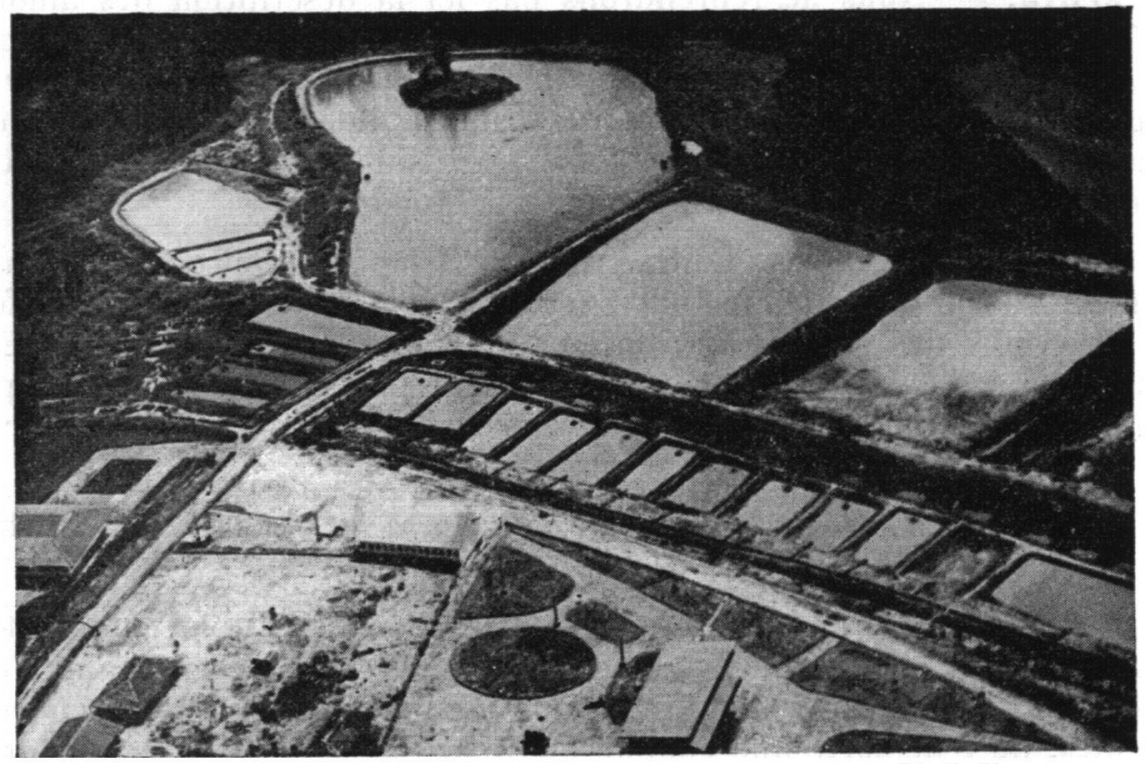

Photo Charpy.

Fig. 1. - Pisciculture de la Djoumouna (Brazzaville).

vue partielle.

Il existe un certain nombre d'autres caractères plus apparents pour distinguer ces deux espèces.

Tilapia melanopleura a la partie inférieure de la nageoire caudale plus claire que la partie supérieure ; Tilapia macrochir possède une nageoire caudale de couleur uniforme, mais son extrémité postérieure est frangée de rouge.

Tilapia macrochir a le dessous de la tête jaunâtre (cette coloration disparaît chez les individus de plus de 18 à $20 \mathrm{~cm}$.). Tilapia melanopleura n'a jamais le dessous de la tête jaunâtre ; il présente généralement une coloration rougeâtre.

Les nageoires anales et ventrales de Tilapia melanopleura sont rougeâtres alors que celles de Tilapia macrochir sont grises.

Caractores soxuols. - La différentiation des sexes est assez aisée chez les individus matures, grâce à la fente transversale de l'orifice génital femelle. Celle-ci n'apparaît qu'au moment de la première ponte; 
une détermination trop précoce risque de ne diagnostiquer que des mâles. Cette fente perpendiculaire à l'axe du corps de la femelle est portée par un très court pédoncule tronconique.

L'orifice uréno-génital du mâle se présente sous la forme d'un minuscule point porté par un court pédoncule conique. Au moment de la période de fraie, ce pédoncule se prolonge souvent par des filaments plus ou moins longs.

Fraie. - Nous ne reprendrons pas ici la description des amours du Tilapia qui a été faite par le Dr. DE Bont. Rappelons seulement que nos deux espèces sont monogames, que Tilapia melanopleura construit dans les digues, presque au niveau de l'eau, un nid constitué de plusieurs trous accolés et que Tilapia macrochir trace sur le fond de l'étang, sous 50 centimètres d'eau environ, un nid en étoile.

Sous le climat de l'A. É. F., ces deux Tilapia se reproduisent toute l'année à raison d'une ponte toutes les six à sept semaines, soit huit fois par an. Dans des climats à variations de température plus accentuées, comme celui d'Élisabethville au Congo belge, les Tilapia ne fraient pas pendant les quatre mois de saison fraîche.

Le Dr. et Mme DE BoNT, en disséquant des femelles mûres, ont compté environ 1.500 œufs chez Tilapia macrochir, 5.000 à 7.000 chez Tilapia melanopleura.

Par suite du grand nombre de pontes il est possible qu'on ait à Brazzaville des chiffres un peu inférieurs ; mais ce qui est certain, c'est qu'il disparaît un grand nombre d'œufs et d'alevins dans les premières semaines, de telle sorte que chaque ponte ne donne pas plus de quelques centaines d'alevins d'un mois. Nous pensons que la principale cause en est la pauvreté planctonique de nos eaux. Cette mortalité des jeunes alevins est finalement une excellente chose puisque, comme nous le verrons, ceux-ci sont exclusivement planctophages pendant les premières semaines et que leur croissance individuelle est d'autant plus rapide qu'ils sont moins nombreux à l'unité de surface.

Croissance. - Nous avons suivi pendant près de deux ans quatre pontes de Tilapia melanopleura et trois de Tilapia macrochir, afin d'établir leur courbe de croissance et de noter leur âge et leur taille de maturité sexuelle. Sauf une que nous signalerons au passage, toutes ces populations ont été placées dans des bassins d'un are environ jusqu'à l'àge de 9-10 mois et ensuite dans des bassins de 2 a. 5 environ. Leur nourriture artificielle n'a malheureusement pas été très régulière; elle est passée en moyenne de 500 grammes par jour au début à 1.300 grammes vers la fin et a été composée de produits les plus divers : son de paddy, farine de manioc, manioc écrasé, feuilles de manioc et de bananiers, etc.

Les tableaux ci-après donnent, pour chacune des deux espèces et pour chaque population, le nombre d'alevins vivant ensemble, leur taille et leur poids moyens à différents âges : 
a) Tilapia melanopleura.

$1^{\text {re }}$ popul. $2^{\mathrm{e}}$ popul. $3^{\mathrm{e}}$ popul. $4^{\mathrm{e}}$ popul.

3 mois :

$\begin{array}{llllll}\text { Nombre d'alevins..... } & 92 & 306 & 1.000 & 6.500\end{array}$

Poids moyen........ 16 gr. $5 \quad 7$ grs 3 gr. $2 \quad 2$ grs

Taille moyenne..... 9 cm. $\quad 6 \mathrm{~cm} .5 \quad 4 \mathrm{~cm} .7 \quad 3 \mathrm{~cm}$.

6 mois :

Nombre d'alevins..... $\quad 90 \quad 293 \quad 263 \quad 6.500$

Poids moyen....... 45 grs 28 grs 26 grs 3 gr. 5

Taille moyenne..... $12 \mathrm{~cm}$. $10 \mathrm{~cm} .510 \mathrm{~cm}$. $6 \mathrm{~cm}$.

12 mois :

Nombre d'alevins.... $\quad 80 \quad 105 \quad 192 \quad 440$

Poids moyen....... 88 grs 56 grs 44 grs 16 grs

Taille moyenne...... $15 \mathrm{~cm} .8 \quad 13 \mathrm{~cm} .6 \quad 12 \mathrm{~cm} .8 \quad 8 \mathrm{~cm} .3$

18 mois :

Nombre d'alevins.... $65 \quad 65 \quad 101 \quad 174$

Poids moyen....... 130 gr. 9105 grs 84 grs

Taille moyenne...... $18 \mathrm{~cm} .517 \mathrm{~cm} . \quad 16 \mathrm{~cm}$.

24 mois :

Nombre d'alevins..... $57 \quad 99$

Poids moyen....... 190 grs 162 grs

Taille moyenne...... $20 \mathrm{~cm} .5 \quad 19 \mathrm{~cm} .6$

b) Tilapia macrochir.

$1^{\text {re }}$ popul. $\quad 2^{\mathrm{e}}$ popul. $\quad 3^{\mathrm{e}}$ popul.

3 mois :

Nombre d'alevins.......

Poids moyen...........

Taille moyenne........

170

$\begin{array}{cc}135 & 260 \\ 20 \mathrm{grs} & 8 \mathrm{grs} \\ 9 \mathrm{~cm} .3 & 7 \mathrm{~cm} .\end{array}$

6 mois :

Nombre d'alevins....... 168

95

200

Poids moyen......... 45 grs

54 grs

$22 \mathrm{grs}$

Taille moyenne...... $13 \mathrm{~cm}$.

$13 \mathrm{~cm} .4 \quad 10 \mathrm{~cm}$.

10 mois :

Nombre d'alevins. ......

159

$104 \mathrm{gr} .3$

77

176

Poids moyen...

$17 \mathrm{~cm} .2$

83 grs

$39 \mathrm{gr}$. 4

Taille moyenne........

$16 \mathrm{~cm} .6$

$13 \mathrm{~cm} .1$ 
18 mois :

$1^{\text {re }}$ popul. $\quad 2^{e}$ popul. $\quad 3^{\mathrm{e}}$ popul.

Nombre d'alevins.......

Poids moyen...........

Taille moyenne........

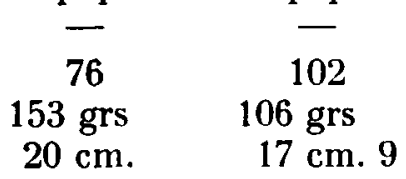

Contrairement aux autres, la première population de macrochir a été conduite presque continuellement dans un étang de 9 ares.

Les très grandes différences présentées par les croissances moyennes de ces diverses populations sont expliquées par le nombre d'alevins existant à l'unité de surface. Le caractère essentiellement planctophage des alevins de Tilapia, lié à la pauvreté des eaux de la station de la Djoumouna $\left(p \mathrm{H}: 5,9\right.$; alcalinité totale en $\mathrm{cm}^{3}$ d'acide $\mathrm{N} / 10$ par litre : 0,3 ; dureté totale en $\mathrm{CaO}$ par litre : $4 \mathrm{mmgr}$.) empêche leur croissance normale dès qu'ils dépassent une certaine densité à l'unité de surface; 100 à 200 alevins du même âge à l'are d'étang paraît être un maximum pour leur assurer un développement correct. Il apparaît que le retard pris pendant les premières semaines ou les premiers mois n'est pas rattrapé ensuite quoique, comme nous le verrons plus loin, les adultes profitent énormément de la nourriture artificielle qu'on leur distribue.

Il semble que chaque classe d'âge d'alevins de Tilapia se comporte en étang comme si elle était seule, ce qui a fait préconiser au Dr. DE BONT la " méthode mixte " où sont représentées dans l'étang toutes les tailles de poissons.

Si la croissaune du Tilapia, est très variable selon la surface d'eau qu'il a à sa disposition, dans une même population les croissances individuelles sont très différentes; les poids individuels s'étagent dès les premiers mois de 1 à 2,5 ; cette proportion se conserve ensuite, semblant confirmer cette hypothèse que le retard pris dans les premières semaines n'est pas comblé par la suite.

La répartition du nombre de poissons par catégorie de taille se présente tout d'abord sous forme d'une courbe classique à un sommet. Très rapidement on voit apparaître deux sommets qui correspondent à une différentiation sexuelle; à un certain moment notre courbe se sépare complètement en deux morceaux.

La croissance des mâles est incomparablement plus rapide que celle des femelles. Nous ne citerons que deux exemples pour fixer les idées :

Dans notre première population de Tilapia melanopleura :

A 16 mois, le poids moyen des 36 mâles est de 126 gr. 2 .

A 16 mois, le poids moyen des 29 femelles est de 87 gr. 4.

A 29 mois, le poids moyen des 30 mâles est de 307 gr. 8 .

A 29 mois, le poids moyen des 27 femelles est de 176 gr. 8 .

Dans notre deuxième population de Tilapia macrochir :

A 14 mois $1 / 2$, le poids moyen des 39 mâles est de 152 grs.

A 14 mois $1 / 2$, le poids moyen des 38 femelles est de 81 grs.

A 18 mois $1 / 2$, le poids moyen des 39 mâles est de 203 grs.

A 18 mois $1 / 2$, le poids moyen des 32 femelles est de 106 grs. 
D'où l'idée - que nous cherchons à mettre actuellement en pratique - d'une culture de màles seuls pour la production de gros poissons.

Les différences de croissance d'un individu à un autre nous ont incité à pratiquer dans notre capital-Tilapia actuel une sélection massale pour isoler les meilleures souches.

Nous avons noté l'apparition de la maturité sexuelle; celle-ci s'échelonne sur près de deux mois pour chacune des populations étudiées. Il semble bien, comme l'avaient noté les chercheurs belges, que cette maturité sexuelle soit liée à une taille donnée plutôt qu'à un âge déterminé. Elle serait à Brazzaville de 12 centimètres pour Tilapia melanopleura

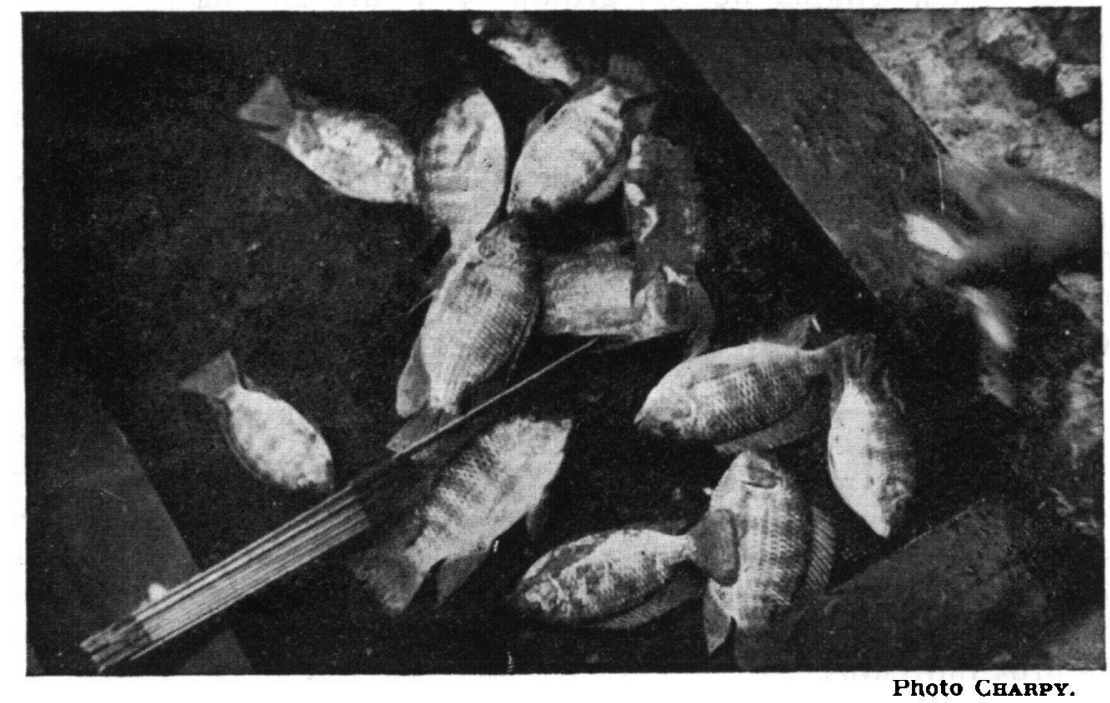

Frg. 2. - Quelques Tilapias produits à la Pisciculture de la Djoumouna.

et 14 centimètres pour Tilapia macrochir, ce qui correspondrait à des poids respectifs de 40 grammes et 60 grammes. Dans les conditions les plus courantes à la Djoumouna, Tilapia melanopleura serait mûr vers 6 à 7 mois et Tilapia macrochir vers 8 mois. Cette taille de maturité aurait tendance à diminuer légèrement chez les individus qui ont eu beaucoup de peine à grandir et vice-versa; c'est ainsi que, dans notre quatrième population de Tilapia melanopleura, elle est apparue vers 11-12 mois alors que la moyenne des plus grands poissons n'était que de 10 centimètres.

A tous les facteurs précédents agissant sur la croissance, nous devons ajouter la richesse planctonique des eaux et la qualité et la quantité de nourriture distribuée; de sorte qu'il est extrêmement difficile de fixer pour chaque âge le poids et la taille du Tilapia moyen.

A Élisabethville, les chercheurs belges ont établi, en opérant sur des populations de 200 alevins du même âge à l'are d'étang, que Tilapia macrochir et Tilapia melanopleura mettaient environ six mois pour atteindre le poids de 50 grammes, qu'ils triplaient leur poids dans les 
quatre mois suivants pour donner à un an des poissons de 180 à 190 grammes mesurant 18 à 19 centimètres.

A Brazzaville, dans les eaux relativement pauvres de cette région et avec une nourriture de qualité moyenne, nous pouvons dire que le Tilapia moyen pèse 25 à 40 grammes à 6 mois et atteint environ 100 grammes à 1 an lorsqu'il est correctement nourri.

La relation entre le poids et la taille est toujours constante :

Un Tilapia de 10 grammes mesure $7 \mathrm{~cm}$. 5 .

Un Tilapia de 30 grammes mesure $11 \mathrm{~cm}$.

Un Tilapia de 100 grammes mesure $17 \mathrm{~cm}$.

Un Tilapia de 150 grammes mesure $20 \mathrm{~cm}$.

Nous avons calculé tous les deux mois pour chaque population le facteur de condition $\mathrm{K}=\frac{100 \mathrm{P}}{\mathrm{L}^{3}}$ ou $\mathrm{P}$ représente le poids en grammes et $\mathbf{L}$ la longueur en centimètres. Ce facteur est constant pour chaque âge et diminue légèrement avec le temps. Il passe de $2,5 \grave{a} 2,1$ chez Tilapia melanopleura et de 2,1 à 1,8 chez Tilapia macrochir entre six et dix-huit mois.

Cette étude sommaire de la croissance nous montre le grand défaut du Tilapia comme poisson de pisciculture : sa petite taille. Cela n'a pas actuellement une grande importance, puisque le premier but à réaliser est de produire du $\mathrm{kg}$. de poisson au meilleur prix pour des populations privées de nourriture carnée. Mais plus tard il nous faudra sans aucun doute soit chercher d'autres espèces de poissons à cultiver, soit améliorer par sélection les deux Tilapia utilisés actuellement, soit mettre au point des méthodes de production à plus faible rendement, mais ne donnant que de gros individus.

Nourrifure. - Le mélange des deux espèces en bassin de pisciculture est basé sur leurs caractéristiques alimentaires différentes. Une étude très précise a été effectuée par les chercheurs belges en analysant des contenus stomacaux de plusieurs centaines de Tilapia de toutes tailles.

Tilapia melanopleura est caractérisé par un régime phytophage très accusé; il suffit de voir comment il se jette sur tous les produits végétaux que l'on peut lui distribuer. On l'utilise même pour nettoyer des pièces d'eau enherbées; mais il est également carnivore à l'occasion.

Tilapia macrochir profite de tous les déchets qu'il peut trouver à sa disposition : petits débris végétaux, larves d'insectes, produits farineux, etc., c'est un omnivore microphage.

Comme l'ont noté les chercheurs belges, les alevins des deux espèces sont dans leur tout jeune âge exclusivement planctophages, mais, dès la taille de 3 centimètres, les jeunes melanopleura viennent, comme leurs parents, déchiqueter les feuilles de manioc ou de bananiers qui leur ont été jetées.

On a remarqué que les Tilapia s'habituaient à une nourriture déterminée au détriment d'une autre; c'est ainsi que nous avons vu des étangs 
nourris avec des fougères où les poíssons dédaignaient les feuilles de manioc dont leurs parents ètaient si friands.

Nous verrons, dans la suite de đet exposé, que la pisciculture du Tilapia n'est vraiment intéressante que si l'on nourrit les étangs. Tout leur est bon; le Tilapia est le meilleur transformateur de déchets ou de sous-produits que l'on connaisse actuellement. Cette nourriture est fonction des disponibilités locales. Elle comprendra naturellement tous les produits végétaux qui existent sur place : feuilles de manioc, de bananier, de légumes, herbe à éléphant, etc. Il n'est pas nécessaire de la hacher, le Tilapia s'en charge et ronge même l'écorce des tiges. Tous les déchets domestiques sont utilisés : non seulement les restes de manioc ou de légumes, mais les peaux de banane, les oranges pourries, etc. Les sous-produits industriels : son de riz, tourteaux d'arachide, déchets d'huilerie de palme et peut-être même les graines de coton ou les parches de café sont transformables en Tilapia. Nous verrons, dans la suite de cet exposé, la valeur de certaines de ces nourritures.

L'équilibre entre les deux espèces est réalisé automatiquement d'après la nourriture qu'on leur distribue. Un comptage a été effectué parmi les poissons de plus de 50 grammes venant de trois bassins nourris pendant quatre mois avec la même quantité de nourriture composée de feuilles de manioc pour le premier, de tourteau d'arachide pour le second et d'un mélange des deux pour le troisième.

Tilapia macrochir et Tilapia melanopleura adultes étaient respectivement représentés par les proportions $10 \%$ et $66 \%, 24,1 \%$ et $13,8 \%$, $22,5 \%$ et $25,2 \%$.

Sur douze bassins nourris pendant un an avec des feuilles de manioc et des termitières et chargés avec des proportions variables des deux espèces, nous avons eu à la vidange un pourcentage constant de $4 \%$ d'adultes macrochir contre $31 \%$ d'adultes melanopleura, le reste étant constitué par des alevins des deux espèces d'un poids inférieur à 50 grammes.

Dans la pisciculture telle qu'on la pratique en Afrique Centrale les deux espèces sont intéressantes. Tilapia melanopleura prendra la première place dans la plupart des bassins familiaux dont une grande partie de la nourriture artificielle est apportée sous forme de végétaux; mais Tilapia macrochir, si on peut lui apporter des produits farineux, donnera des poissons plus gros et de croissance plus rapide. Le dosage des deux espèces, s'il est délicat à réaliser au moment de la première mise en charge, s'établira automatiquement par la suite d'après la nourriture que ces poissons auront à leur disposition.

\section{L'EXPLOITATION DES ETANGS DE PISCICULTURE DE THAPIA}

Lorsque nous avons commencé nos études sur le Tilapia à la station de Pisciculture de la Djoumouna nous connaissions un certain nombre de principes essentiels mis à jour par les chercheurs belges.

Les caractères généraux de la "méthode mixte" du Dr. DE BonT 
ont été exposés par M. le Conservateur L.emasson dans le $n^{\circ} 173 \mathrm{du}$ Bulletin Français de Pisciculture :

Alevinage et production dans un même étang.

Mise en charge initiale très forte.

Élevage en mélange de poissons de toutes tailles.

Fumure et alimentation artificielle aussi intense que possible.

Récolte à intervalle régulier (semaines, mois) des poissons ayant dépassé la taille correspondant à celle de la reproduction.

Vidange des étangs chaque année pour remise en état, nettoyage, etc., et réempoissonnement avec les poissons pêchés n'ayant pas atteint la taille marchande.

Cette méthode basée sur la culture du poisson de toutes tailles et de tous âges est parfaitement conforme à ce que nous a appris l'étude du Tilapia.

Nous savions qu'on avait obtenu à Élisabethville des rendements allant jusqu'à 9 et 11 tonnes de poisson par hectare et par an.

Les pisciculteurs belges nous avaient dit qu'on devait déverser chaque jour dans l'étang un poids de nourriture sensiblement égal au vingtième du poids du poisson qui s'y trouvait et qu'au total il fallait compter 5 à $7 \mathrm{kgs}$ de nourriture pour fabriquer un $\mathrm{kg}$. Tilapia. La base de la nourriture des étangs d'Élisabethville était les brisures de minoterie.

Nous avions vu nous-mêmes, en 1952, des étangs africains du centre $\mathrm{du}$ Congo belge nourris avec des feuilles de manioc, des patates douces, des termitières, des déchets de manioc, etc.

Nous avions enfin assisté à l'écrémage de grands étangs de production par des filets maillants en nylon qui capturaient des poissons de 150 à 200 grammes.

Nous allons commencer par expliquer ici comment, partant de ces données, nous sommes parvenus à nos idées actuelles sur le Tilapia.

Pour pouvoir distribuer chaque jour un poids de nourriture égal au vingtième du poids du poisson qui se trouve dans l'étang, il fallait connaître la courbe de croissance d'une population de Tilapia en étang; elle se présente, tout au moins au début, sous une forme logarithmique. Si $P$ est le poids du poisson placé dans l'étang, on doit lui distribuer quotidiennement, pendant les premiers jours, un poids de nourriture égal à $\frac{\mathrm{P}}{20}$ soit $\frac{30 \mathrm{P}}{20}$ en un mois; ces $\frac{3}{2} \mathrm{P}$ kgs de nourriture permettent de fabriquer $\frac{3}{2} \mathrm{P} \times \frac{1}{5}=0,3 \mathrm{P}$ kgs de poisson. En continuant le raisonnement pendant les mois suivants, on voit que le poids de poisson $Y$ au mois $x$ est donné par la formule $\mathrm{Y}=\mathrm{P} 1,3 x$.

Il est bien certain que cette formule n'est valable que dans certaines limites, car il arrivera un moment où l'étang atteindra sa charge maxima et où la courbe de croissance prendra une allure asymptotique. Nous pensions à priori qu'elle pourrait être vérifiée pour une charge passant de $100 \mathrm{kgs}$ à 2 tonnes à l'hectare, ce qui nous permettrait de déduire 
le poids de nourriture ì distribuer chaque mois. Nous pensions que le coefficient 1,3 serait à modifier légèrement selon la richesse de l'eau et la qualité de nourriture artificielle.

D'après la formule ci-dessus, $100 \mathrm{kgs}$ de poissons placés à l'hectare d'étang devaient donner :
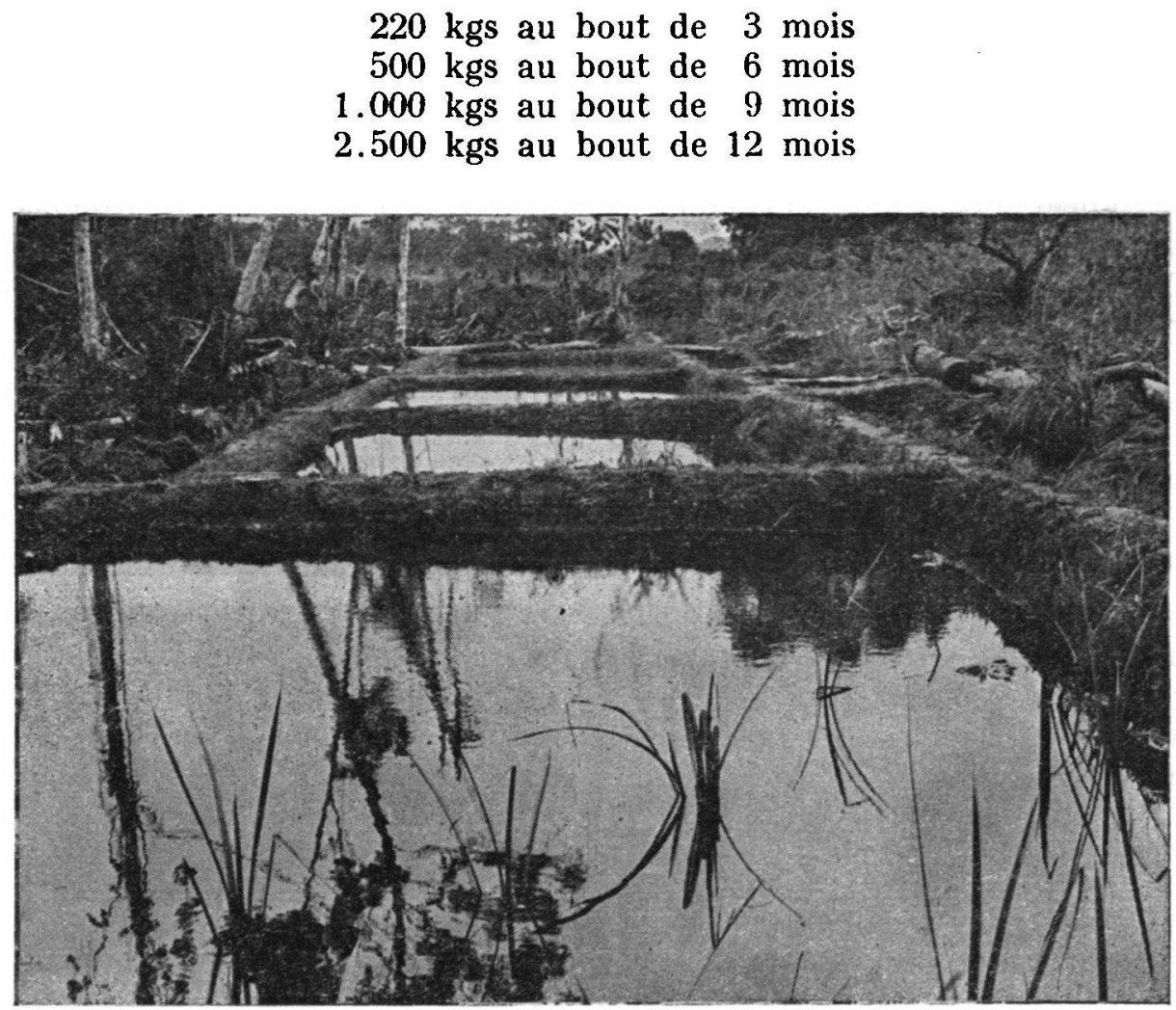

FIG. 3. - Etangs de pisciculture familiale.

Photo Crarpt.

Région du Pool (Moyen Congo).

Nous avons donc essayé de la vérifier ou de voir dans quel sens elle était fausse, en vidant tous les trois mois un étang chargé au départ avec $100 \mathrm{kgs}$ de poisson par hectare et nourri comme nous venons de l'indiquer avec de la farine de manioc et de mil, du manioc rouï, du son de riz et des feuilles de bananiers; nous avons obtenu :
$225 \mathrm{kgs}$ à l'hectare au bout de 3 mois
$500 \mathrm{kgs}$ à l'hectare au bout de 6 mois
$1.000 \mathrm{kgs}$ à l'hectare au bout de 9 mois
$2.000 \mathrm{kgs}$ à l'hectare au bout de 12 mois
$2.353 \mathrm{kgs}$ à l'hectare au bout de 14 mois $1 / 2$

La courbe réelle qui se superposait à la courbe théorique jusque vers $1.200 \mathrm{kgs} / \mathrm{ha}$. se trouvait ensuite un peu en dessous pour tendre vers une asymptote avant $2.500 \mathrm{kgs}$. 
Un certain nombre d'autres étangs chargés avec des poids variables, exploités pendant des durée svariables, mais nourris d'après cette formule, nous avaient donné des chiffres concordant parfaitement avec les expériences précédentes. Nous en avions donc déduit la courbe de croissance d'une population de Tilapia à l'hectare d'étang correctement nourri et nous ajoutions que les eaux de la Djoumouna avaient une capacité maximum de $2.500 \mathrm{kgs} / \mathrm{ha}$. correspondant à l'asymptote de la courbe.

Pour avoir le meilleur rendement des étangs il fallait conduire une population de Tilapia au moment où son taux d'accroissement était maximum. Théoriquement, en chargeant l'hectare d'étang avec $1.000 \mathrm{kgs}$ et en le vidant au bout de trois mois on retirerait $2.000 \mathrm{kgs}$ soit un gain d'une tonne par trimestre, c'est-à-dire 4 tonnes par an. Mais pour ne pas multiplier les vidanges, nous conseillions de charger à $500 \mathrm{kgs}$ (soit $1 / 5^{\mathrm{e}}$ de la capacité maximum) et de vider au bout de six mois pour obtenir finalement un rendement théorique de 3 tonnes par hectare et par an. On exploitait donc l'étang entre le cinquième et les quatre cinquièmes de sa capacité maximum.

En admettant que la capacité maximum d'un étang était variable selon la qualité des eaux et selon la qualité de la nourriture il nous suffisait de la déterminer pour chaque cas particulier afin de connaître les limites optima d'exploitabilité. Nous pensions alors que la nourriture artificielle ne pourrait être utilisée que dans la mesure où notre population de Tilapia disposerait d'une certaine quantité de nourriture naturelle. Celle-ci étant fixé dans une eau déterminée (compte tenu des façons culturales possibles : assec, fumure, engrais), il semblait normal de pouvoir définir une capacité maxima des étangs et de penser qu'un excès de nourriture artificielle n'aurait qu'une action extrêmement minime sur cette capacité.

Le premier but que nous nous étions assigné semblait être atteint puisque nous avions établi la courbe de croissance d'une population de Tilapia à l'hectare d'étang.

Des quelques étangs qui avaient été pêchés à la ligne pendant la période d'exploitation nous avions conclu que, si l'on reste dans certaines limites, le poisson retiré par des pêches intermédiaires est immédiatement remplacé; la vidange de l'ètang donne le même résultat qu'il ait été "écrémé " ou non. Si nous étions en accord avec le Dr. DE Bont sur le principe des pèches intermédiaires, nous ne l'étions plus quant aux modalités de cet écrémage pour des raisons biologiques et techniques :

Le fait d'enlever les poissons au fur et à mesure qu'ils atteignent une taille donnée consiste à faire une sélection à rebours et à ne laisser comme géniteurs dans l'étang que les poissons de mauvaise venue. D'autre part, dans les eaux pauvres en plancton comme celles de la Djoumouna et de la plupart des régions d'A. É. F., le Tilapia donne trop d'alevins qui se nuisent mutuellement; on a donc intérèt à retirer une partie des jeunes au fur et à mesure de leur production pour laisser plus d'espace aux autres. 
Enfin, la sélection des poissons d'une taille déterminée par les pèches intermédiaires ne peut être faite jusqu'ici que par des filets maillants en nylon, les Tilapia ne se laissant pas prendre dans les filets en coton. Si un tel procédé peut être, à la rigueur, concevable dans de grandes exploitations industrielles, il est beaucoup trop coûteux en pisciculture familiale.

C'est pourquoi nous conseillons plus simplement d'enlever chaque semaine un certain nombre de kgs de poisson sans tenir compte de la taille.

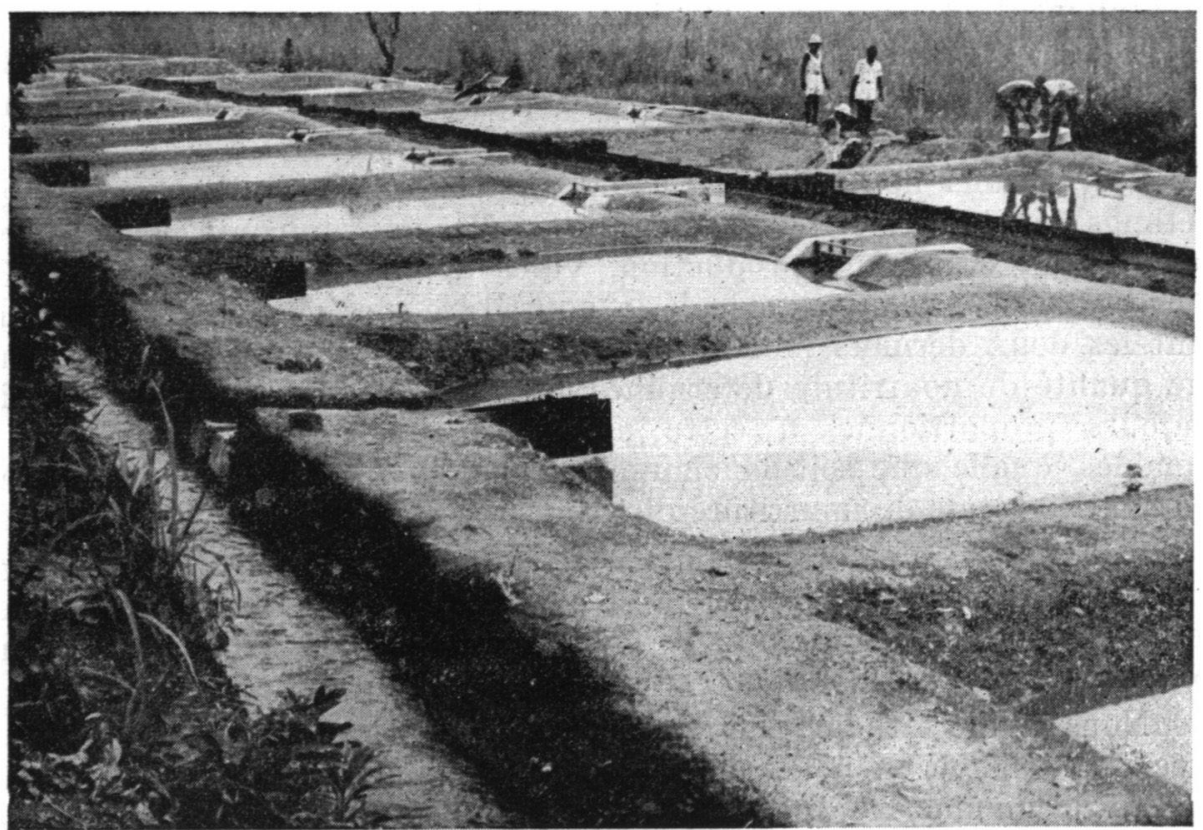

Fia. 4. - 86rie de potits stang: d'un are.

Photo Charpy.

Station de la Landjia, à BANaur.

Ce problème des pêches intermédiaires est finalement le plus délicat à résoudre dans la pisciculture du Tilapia. Dans les petites exploitations familiales, les deux seuls modes de pêches efficaces sont la ligne et l'éperviér. Le rendement de l'épervier reste constant à condition d'avoir repéré l'emplacement du bassin où se trouvent les poissons.

Si au début les poissons mordent très facilement à l'hameçon, il arrive un moment où ils s'en lassent et où le rendement baisse considérablement. C'est ainsi qu'un étang de 37 ares qui renfermait $415 \mathrm{kgs}$ de poissons (1.100 kgs/ha.) était pêché à la ligne tous les quinze jours par une vingtaine de pêcheurs pour retirer chaque fois environ $30 \mathrm{kgs}$, c'est-à-dire un peu moins que ce qui était considéré comme l'accroissement de la population pendant ce temps. Alors que les premières fois ces trente kgs étaient retirés en trente heures de pêche, au bout de deux mois, quarante heures suffisaient à peine pour retirer $7 \mathrm{kgs}$. Par contre, des petits étangs d'un are, pèchés à la ligne tous les quinze jours à raison 
de 150 grs, 300 grs et 450 grs chaque fois, ont donné des résultats assez réguliers.

Dans les grands bassins de pisciculture industrielle, tout ce que nous avons essayé jusqu'ici : senne, filet maillant en coton, carrelet, tramail, etc., a échoué.

Les travaux réalisés depuis un an sur la Station de Pisciculture de la Djoumouna nous ont éclairés sur un nouvel aspect du Tilapia, qui permet maintenant de le considérer comme l'un des meilleurs transformateurs de déchets ou de sous-produits qui soit connu et qui fait rapprocher sa culture de celle de la Truite, en ce sens qu'il transforme intégralement et très rapidement en kgs de poisson la nourriture artificielle qui lui est distribuée. L'eau, outre qu'elle est la source essentielle de nourriture de tout jeune alevin, ne servirait ensuite que de support et de source d'oxygène.

Ces notions nouvelles nous sont apparues à la suite de vidange d'étangs traités à très courte révolution; elles ont été confirmées par les soixante-dix étangs de production, vidés depuis le $1^{\text {er }}$ Janvier 1953, pour lesquels nous avons calculé la nourriture moyenne distribuée pendant les deux derniers mois de charge. Nous avons constaté que, pour une qualité de nourriture déterminée, le poids obtenu à la vidange était toujours proportionnel à cette nourriture journalière des dernières semaines, quelle que soit la charge initiale, la durée d'exploitation et la quantité totale de nourriture distribuée pendant cette mise en charge.

Nous avons alors été amenés à définir une "capacité " des étangs pour une distribution journalière et constante de nourriture artificielle. Cette " capacité " représente le poids maximum de Tilapia que peut contenir un hectare d'étang traité dans ces conditions.

Nous avons étudié plus spécialement deux types de nourriture; avec un mélange en poids de $2 / 3$ de tourteau d'arachide et de $1 / 3$ de feuilles de manioc la capacité de l'hectare d'étang à la Djoumouna est de :

$960 \mathrm{kgs}$ pour une distribution de $15 \mathrm{kgs}$ de nourriture par ha. et par jour $1.300 \mathrm{kgs}$ pour une distribution de $25 \mathrm{kgs}$ de nourriture par ha. et par jour $1.450 \mathrm{kgs}$ pour une distribution de $30 \mathrm{kgs}$ de nourriture par ha. et par jour $2.000 \mathrm{kgs}$ pour une distribution de $50 \mathrm{kgs}$ de nourriture par ha. et par jour $2.750 \mathrm{kgs}$ pour une distribution de $75 \mathrm{kgs}$ de nourriture par ha. et par jour $3.080 \mathrm{kgs}$ pour une distribution de $100 \mathrm{kgs}$ de nourriture par ha. et par jour

Avec un mélange dans les mêmes proportions de son de riz et de feuilles de manioc la capacité serait environ de :

$750 \mathrm{kgs}$ de poisson pour une distribution de $25 \mathrm{kgs} / \mathrm{ha}$./jour

$1.150 \mathrm{kgs}$ de poisson pour une distribution de $50 \mathrm{kgs} / \mathrm{ha}$./jour

$1.400 \mathrm{kgs}$ de poisson pour une distribution de $75 \mathrm{kgs} / \mathrm{ha} . / \mathrm{jour}$

$1.650 \mathrm{kgs}$ de poisson pour une distribution de $100 \mathrm{kgs} / \mathrm{ha}$./jour

$2.150 \mathrm{kgs}$ de poisson pour une distribution de $150 \mathrm{kgs} / \mathrm{ha}$./jour

$2.550 \mathrm{kgs}$ de poisson pour une distribution de $200 \mathrm{kgs} / \mathrm{ha}$./jour

Nous nous sommes rendus compte, enfin, que cette capacité est atteinte extrêmement rapidement si on prend soin aussitôt, après la 
mise en charge de l'étang, de distribuer chaque jour la dose correspondante de nourriture. Nous avons fait passer en quatre mois, 500 kgs de Tilapia à $3.050 \mathrm{kgs}$ dans un étang d'un hectare, en distribuant chaque jour $99 \mathrm{kgs}$ de tourteau d'arachide et de feuilles de manioc; il est probable que cette charge était déjà atteinte depuis plusieurs semaines quand nous avons vidé l'étang. Treize jours après avoir placé $500 \mathrm{kgs}$ de poisson dans un hectare d'étang nourri avec $50 \mathrm{kgs}$ de tourteau d'arachide et de feuilles de manioc par jour, nous en avons retiré $780 \mathrm{kgs}$.

La transformation de la nourriture artificielle en kgs de poisson se fait naturellement avec un coefficient variable selon la qualité de cette nourriture. Si on appelle " quotient nutritif " le nombre de kgs de nourriture nécessaire pour former un $\mathrm{kg}$. de Tilapia, celui-ci serait :

1,5 à 4 pour un mélange tourteau d'arachide, feuilles de manioc.

6 à 12 pour un mélange de son de riz, feuilles de manioc.

12 à 20 pour les feuilles de manioc seules.

En effet, le prix de revient de cette transformation pour une nourriture déterminée, c'est-à-dire le quotient nutritif est d'autant plus élevé que l'on désire obtenir un plus gros rendement à l'hectare d'étang. C'est ainsi qu'avec le mélange tourteau d'arachide, feuilles de manioc le prix du kilogramme de Tilapia serait celui de :

$2 \mathrm{kgs} 500$ de nourriture si on veut faire contenir $1.300 \mathrm{kgs}$ à l'hectare (correspondant à une distribution de $25 \mathrm{kgs} / \mathrm{ha}$./jour de nourriture).

$3 \mathrm{kgs} 100$ de nourriture si on veut faire contenir $2.500 \mathrm{kgs}$ à l'hectare (correspondant à une distribution de $60 \mathrm{kgs} / \mathrm{ha}$./jour de nourriture).

$3 \mathrm{kgs} 300$ de nourriture si on veut faire contenir $3.000 \mathrm{kgs}$ à l'hectare

(correspondant à une distribution de $95 \mathrm{kgs} / \mathrm{ha}$./jour de nourriture).

Lorsque nous nourrissions nos étangs selon la courbe progressive de nourriture, nous avions en réalité chaque mois dans notre bassin la capacité de celui-ci pour la nourriture distribuée à ce moment-là. Les quotients nutritifs étaient généralement supérieurs à ce qu'ils devaient être parce que nous perdions du temps dans la fabrication de notre poisson et qu'une partie de la nourriture distribuée servait uniquement à l'entretien du stock du poisson existant.

La majorité des chiffres énoncés ci-dessus a été obtenue au cours d'expériences qui n'avaient pas pour but de prouver les idées auxquelles nous sommes parvenus actuellement. Afin de donner toute la rigueur possible à notre démonstration, nous venons de mettre en route un protocole d'essais destinés d'une part à établir la courbe de croissance d'une population de Tilapia nourris à une dose constante de nourriture et à prouver que la capacité est atteinte en trois à quatre mois à partir d'une charge égale au cinquième de cette capacité et, d'autre part, à déterminer exactement les capacités relatives à chaque dose journalière des principales nourritures intéressantes sur le plan local d'A. É. F. et à en déduire les quotients nutritifs correspondants.

Nous pensons pouvoir mettre à la disposition des pisciculteurs des tables de nourriture qui leur permettront, d'après le prix de celle-ci, de déterminer quel est le mode de transformation le plus rentable pour 
eux : faibles rendements avec quotient nutritif minime ou bien hauts rendements avec quotient nutritif plus élevé.

Quand on sait que la capacité de l'hectare d'étang non nourri artificiellement est d'environ $250 \mathrm{kgs}$ à la Station de Pisciculture de la Djoumouna et qu'avec des distributions journalières de $100 \mathrm{kgs}$ de tourteau d'arachide et de feuilles de manioc nous avons élevé cette capacité à plus de 3 tonnes, nous voyons le rôle très faible de l'eau comme nourriture. Il est fort probable que des variations dans la qualité de cette eau n'auraient que des incidences assez minimes sur le rendement des étangs; c'est le sens des essais qui viennent d'être commencés dans les régions calcaires du Niari à 150 kilomètres de Brazzaville. Cela expliquerait également que les différents essais de fumure organiques effectués sur la station de la Djoumouna n'aient donné que de faibles augmentations de rendement.

Signalons enfin que, dans une ferme expérimentale du Service de l'Agriculture où l'eau présente les mêmes caractéristiques chimiques qu'à la Djoumouna, on a retiré plus de $90 \mathrm{kgs}$ de Tilapia d'un are d'étang qui avait reçu les déchets de la porcherie.

D'après ce que nous venons de voir il serait préférable, pour obtenir le rendement maximum, de vider l'étang dès que la capacité est atteinte, c'est-à-dire tous les trois ou quatre mois. Ces vidanges fréquentes exigent beaucoup de manipulations et apportent, chaque fois, au pisciculteur un gros stock de poissons dont la conservation est délicate; c'est pourquoi il est préférable, tout en continuant à distribuer le même poids de nourriture artificielle, de retirer chaque mois, par des pêches intermédiaires, le poids de poisson qui peut être reformé au cours des trente jours qui suivent. Nous allons chercher à le déterminer, mais nous pensons qu'il doit être au moins égal au cinquième de la capacité. Le problème technique des pêches intermédiaires se pose donc toujours.

Ces notions nouvelles modifient assez peu le mode d'exploitation des étangs de pisciculture à Tilapia que nous avions énoncé en 1954 dans les publications destinées à l'A. E. F. :

Une fois déterminé la capacité de l'étang pour la quantité de nourriture qu'il sera possible de distribuer chaque jour, on met en charge avec un poids de poisson sensiblement égal au cinquième de cette capacité.

Pendant trois mois on le neurrit sans y toucher pour laisser le bassin atteindre sa pleine charge.

Pendant les trois mois suivants on l'exploite intensivement par des pêches intermédiaires : ligne, épervier, etc., pour retirer mensuellement ce qui a été placé initialement. Six mois après la charge, on le vide et on recommence la même opération après l'avoir réensemencé avec des poissons de toutes tailles. 